\title{
Química Analítica: Una Oportunidad para la Inclusión de Aspectos Sociales y Culturales en Ingeniería Química
}

\author{
Ana M. Martín ${ }^{1}$ y Cristina Speltini ${ }^{2}$ \\ (1) Universidad de Buenos Aires, Facultad de Ingeniería, Departamento de Química, Paseo Colón \\ 850. Buenos Aires-Argentina. (e-mail: amartin4c@gmail.com) \\ (2) Universidad Tecnológica Nacional, Facultad Regional Avellaneda, Departamento de Ingeniería \\ Química. Av. Mitre 750. Avellaneda-Argentina. (e-mail: cspeltini@gmail.com)
}

\begin{abstract}
Resumen
Este trabajo se propone identificar y analizar que aspectos sociales y culturales pueden ser tratados durante el desarrollo de una asignatura con un claro sesgo técnico, como lo es Química Analítica. Para ello se realizó un estudio comparativo del curriculum de Química Analítica para la carrera de Ingeniería Química de cinco universidades, cuatro argentinas y una española. Se utilizaron como registros las entrevistas realizadas a los profesores y el material bibliográfico por ellos aportados. Del análisis de las entrevistas surgieron cuatro categorías: concepto de análisis químico, concepto de muestra, interacción ciencia-tecnología-sociedad-ambiente, y aspectos sociales. Los programas del curso de química analítica no incluyen materia ni objetivos relacionados con los aspectos sociales, pero los docentes entrevistados son concientes de la necesidad de utilizar el enfoque ciencia-tecnología-sociedad-ambiente para una adecuada formación de los futuros profesionales Ingenieros Químicos.
\end{abstract}

Palabras clave: química analítica, curriculum, sociedad, ambiente, ingeniería química

\section{Analytical Chemistry: An Opportunity to Include Social and Cultural Aspects in Chemical Engineering}

\begin{abstract}
The purpose of this article is to analyze and identify the type of social and cultural aspects that can be included during the teaching of a technical course such as Analytical Chemistry. To this end, a comparative analysis of the Analytical Chemistry curriculum for the Chemical Engineering major in five universities, four from Argentina and one from Spain, was done. Interviews to professors and bibliographic material provided by them were used in the study. From the analysis, four main aspects are included in the courses: chemical analysis, sampling criteria and the interrelation science-technology-society-environment and social aspects. The programs of analytical chemistry courses do not include this aspects and it is necessary to include aspects related to the relation science-technology-society-environment to achieve an appropriate formation of the future chemical engineers.
\end{abstract}




\section{INTRODUCCIÓN}

Ciencia y tecnología constituyen los pilares de la ingeniería. Sin embargo, la percepción pública de la ciencia y de la tecnología resulta ambivalente. La proliferación de mensajes contrapuestos de tipo optimistas y catastróficos, en torno al papel de estos saberes en las sociedades actuales, ha llevado a que muchas personas no tengan muy claro qué es la ciencia y qué es la tecnología y cuáles son sus respectivos roles en la sociedad. Según Corchuelo (2004) la concepción clásica de las relaciones entre la ciencia y la tecnología con la sociedad, es una concepción triunfalista, según la cual "especialistas" producen ciencia, cuyas aplicaciones tecnológicas conllevan al desarrollo y al bienestar social. Sin embargo, el mundo ha sido testigo de una sucesión de desastres relacionados con cuestiones científico tecnológicas, incrementados desde la segunda Guerra Mundial, por ejemplo, vertidos de residuos contaminantes (Bophal en India), accidentes nucleares (Chernobyl en Rusia), envenenamientos farmacéuticos (diversos pueblos en África), derrames de petróleo (Buque Nelson Valdez) entre otros muchos otros. Estos hechos confirman la necesidad de revisar la política científico - tecnológica de un país y su relación con la sociedad, reflexionando acerca de los procesos de formación de recursos humanos.

El conocimiento tecnológico y el conocimiento científico se encuentran relacionados; son campos disciplinares interdependientes y autónomos. La Ciencia explica y predice en tanto que la Tecnología crea artefactos y diseños. El producto característico de la Ciencia es el conocimiento de la naturaleza, basado en una teoría del mundo material, mientras que el de la Tecnología está constituido por productos materiales, conocidos como artefactos en sentido antropológico y "hardware" en sentido ingenieril. La relación Ciencia - Tecnología - Innovación no es lineal ni responde necesariamente a un orden jerárquico; implica una compleja trama de influencias recíprocas. Los cambios técnicos o científicos se desarrollan en contextos definidos. Existe un contexto interno, que es por ejemplo el laboratorio, la empresa o el taller donde se llevan a cabo las actividades, y un contexto externo, el social, que se relaciona con las dimensiones amplias (políticas, económicas, culturales, ambientales, etc.) Ambos contextos no están aislados, interactúan y son decisivos para las actividades científicas y tecnológicas (Speltini y Cornejo, 2005).

La expresión "ciencia, tecnología, sociedad y ambiente" (CTSA) suele definir un ámbito de trabajo académico cuyo objeto de estudio está constituido por los aspectos sociales de la ciencia y la tecnología, tanto en lo que concierne a los factores sociales que influyen sobre el cambio científico - tecnológico, como en lo que atañe a las consecuencias sociales y ambientales (Corchuelo, 2004). Los enfoques CTSA no necesitan nuevos y complejos trayectos curriculares para su implementación. Se insertan bajo modalidades tan accesibles como pequeños debates, por ejemplo, sobre la contaminación del agua, o un análisis sobre los efectos y las responsabilidades del manejo de los residuos y rellenos sanitarios de una ciudad, etc. De esta manera se pueden tratar en una clase, cuando se presente la oportunidad, o bien se puede proponer explícitamente a los estudiantes analizar las diferentes y variadas interacciones entre la ciencia, la tecnología y la sociedad.

El Informe Grinter (1994), elaborado por la American Society for Engineering Education y publicado en Estados Unidos en 1955, recomendó que la enseñanza de las ingenierías se sustentara en la obligación de la profesión con la sociedad y en la importancia del desarrollo de los estudiantes como individuos. La obligación de los ingenieros de servir a la sociedad involucra el compromiso de mantener un ambiente saludable y seguro para los seres humanos, sin ataduras económicas. Su actividad tiene un efecto directo sobre el bienestar y seguridad de un gran segmento de la sociedad. Los ingenieros deben trabajar en su arte y decidir cual de las varias posibilidades tecnológicas que ofrece un proyecto, provee la mejor solución a un dado problema. La Ingeniería Química trata sobre el diseño, operación y optimización segura, respetuosa del medio ambiente, la energía eficiente de los procesos que transforman las materias primas hasta los productos útiles que nos ayudan en nuestra vida cotidiana (University of Aberdeen, 2009). La Ingeniería Química es una de las más excitantes y multifacética carrera de nuestro tiempo. Debido a que la Ingeniería Química interactúa con la biología, la química, la ciencia de los materiales, la ingeniería civil y eléctrica y es considerada como la disciplina central de la ingeniería. Las oportunidades de plantear y resolver problemas de interés social, son ilimitadas (MIT, 2008). 
En términos amplios, los ingenieros químicos son responsables de la concepción y diseño de procesos con el propósito de producción, transformación y transportación de materiales. Esta actividad comienza con la experimentación en el laboratorio y continua con la implementación de la tecnología a escala producción (Stanford University, 2008). Dentro del campo de su profesión, el Ingeniero Químico se enfrenta a problemas que exigen conocer especificaciones de las materias primas y de los productos terminados, además necesita identificar las variables del proceso que influyen en la calidad de los materiales. Su función es encontrar y aportar soluciones a los problemas que se presenten en: el diseño y la tecnología para la obtención de nuevos productos, la selección y optimización de las fuentes de energía, la evaluación de la contaminación ambiental, la higiene y seguridad industrial, y el tratamiento de efluentes industriales.

En las áreas de energía, producción de alimentos y medicamentos, y cuidado ambiental, los desafíos a resolver implican conocer cómo se realizan ciertas transformaciones y cómo interactúan las sustancias químicas con los sistemas naturales. Ciertos sectores de la industria, tanto en las actividades básicas como en servicios, requieren Ingenieros Químicos para la implementación de nuevas tecnologías de procesamiento de materiales, con crecientes requisitos de calidad y normalización, y cumplimiento de exigentes acuerdos y legislaciones sobre salud, contaminación ambiental y comercio internacional. Dentro de esta rama de la ingeniería, la Química Analítica debe capacitar a los estudiantes en el conocimiento de la funcionalidad de los laboratorios de análisis para el control de procesos industriales y de las variables propias del ambiente natural, especialmente en el control de efluentes.

Con relación al trabajo en laboratorio, el informe Grinter (1994) sostiene que, se le debe dar al estudiante la oportunidad de observar los fenómenos y encontrar explicaciones, probar teorías y encontrar contradicciones, desarrollar experimentos que produzcan datos e interpretar los resultados. La realización de adecuados experimentos por los mismos estudiantes tiene un gran valor educacional. El arte de las medidas, incluyendo el análisis de exactitud, precisión e incertezas, y la apreciación del grado de exactitud económicamente justificada, junto con la comprensión de métodos estadísticos son elementos esenciales del trabajo de laboratorio. Los informes de laboratorio presentan la oportunidad de desarrollar habilidades para la comunicación escrita, como así también las expresiones gráficas.

Es conveniente que los estudiantes desarrollen los criterios para la selección del método de análisis más adecuado de una muestra problema, conociendo las ventajas y desventajas de cada técnica, sus fuentes de incertezas e interpretando adecuadamente los resultados. Si bien el Ingeniero Químico habitualmente no ejecuta los análisis, es importante que sepa qué análisis solicitar, con qué exactitud y precisión debe requerirlos. Consideramos que para mantener el ritmo de la industria, los ingenieros del mañana no sólo deben ser capaces de diseñar, implementar y operar los procesos cada vez más diversos, sino también deben estar equipados con una capacidad adaptable y base de conocimientos que puedan evaluar los costes reales de las decisiones de inversión social y el medio ambiente natural. (University of Surrey-Guildford. 2009). Este trabajo se propone identificar y analizar si algunos aspectos sociales y culturales como los mencionados anteriormente, pueden ser tratados durante el desarrollo de una asignatura con un claro sesgo técnico, como es Química Analítica.

\section{METODOLOGIA}

En esta investigación se ha seleccionado el enfoque cualitativo porque se orienta a describir e interpretar los fenómenos sociales, y por consiguiente los educativos, y se interesa por el estudio de los significados e intenciones de las acciones humanas desde la perspectiva de los propios agentes sociales. Dentro de la metodología cualitativa se ha seleccionado la modalidad de estudio de caso, con la que se ha tratado de describir una situación particular, clasificando sistemáticamente las características de las variables en juego, y estableciendo las posibles relaciones entre variables. El estudio de casos puede considerarse como una estrategia de diseño de la investigación cualitativa encaminada a la toma de decisiones. Dado que se suelen estudiar casos aislados, no se pueden lograr generalizaciones. Sin embargo, es posible incorporar toda la información obtenida en el estudio de caso, convenientemente clasificada, en una base de datos a la que otros puedan recurrir por información. (Alvarez-Gayou, 2003). 
En el estudio presentado, se ha realizado un análisis comparativo del curriculum de Química Analítica para la carrera de Ingeniería Química de cinco universidades, cuatro argentinas y una española. Se utilizaron como registros entrevistas realizadas a los profesores y material bibliográfico por ellos aportados, entre otros los programas analíticos. Los docentes entrevistados pertenecen a la Universidad de Buenos Aires (UBA), Universidad Nacional del Litoral (UNL), Universidad Tecnológica Nacional Facultad Regional Avellaneda (UTN-FRA), Universidad Nacional del Sur (UNS) y Universidad Autónoma de Barcelona (UAB). Para la identificación de los profesores entrevistados se utilizaron los siguientes códigos: los profesores de la UBA se identifican como $L$ y $B$, el profesor de UNL como $H$, los profesores de UTN-FRA como $C$ y $V$, los de UNS: Q, A y Z, y el de UAB: G.

El análisis de las entrevistas se llevó a cabo utilizando la teoría enraizada de Glaser y Strauss (1968). Ésta se basa en el establecimiento, saturación y reorganización de categorías. En aquellas investigaciones exploratorias en que se desconoce cuales son las variables relevantes, la teoría enraizada es un instrumento eficiente que aporta un camino sistemático para realizar este tipo de tarea. Como herramienta informática se ha empleado el programa Non Numerical Unstructured Data Indexing Searching and Theorizing (NUD+IST), que permite el cruce y triangulación de categorías.

\section{RESULTADOS Y DISCUSIÓN}

Del análisis de las entrevistas, empleando el Programa NUD+IST han surgido las categorías siguientes: a) Concepto de análisis químico; b) Concepto de muestra; c) Interacción CTSA; y d) Aspectos sociales.

No todas las categorías han sido mencionadas por igual, siendo la categoría Análisis Químico la que presenta mayor porcentaje, le siguen las menciones a la relaciones entre Ciencia, Tecnología, Sociedad y Medio Ambiente. En la Tabla 1 se encuentran los resultados.

Tabla 1: Porcentajes de presencia de las categorías en las entrevistas

\begin{tabular}{|l|c|}
\hline Categorías & \% presencia en discursos \\
\hline Concepto de Análisis Químico & $7,5 \%$ \\
\hline Concepto de muestra & $1,3 \%$ \\
\hline Interacción CTSA & $3,5 \%$ \\
\hline Aspectos sociales & $2 \%$ \\
\hline
\end{tabular}

En la Tabla 2 se informan las unidades de texto que emergen del discurso de cada entrevistado, para cada categoría, y se las expresa como porcentajes relativos al propio discurso. Con $\mathrm{N}$ se indican aquellas entrevistas en las que no se ha encontrado la categoría.

Tabla 2: Porcentajes de unidades de textos del discurso de cada entrevistado

\begin{tabular}{|l|c|c|c|c|c|c|c|c|c|}
\hline Categorías & A & B & C & G & H & L & Q & V & Z \\
\hline Concepto de Análisis Químico & $23 \%$ & $5 \%$ & $38 \%$ & $7 \%$ & $6 \%$ & $12 \%$ & $1 \%$ & $4 \%$ & $5 \%$ \\
\hline Concepto de muestra & $8 \%$ & $1 \%$ & $8 \%$ & $1 \%$ & $\mathrm{~N}$ & $\mathrm{~N}$ & $\mathrm{~N}$ & $1 \%$ & $6 \%$ \\
\hline Interacción CTSA & $4 \%$ & $1 \%$ & $26 \%$ & $2 \%$ & $1 \%$ & $4 \%$ & $\mathrm{~N}$ & $1 \%$ & $10 \%$ \\
\hline Aspectos sociales & $8 \%$ & $4 \%$ & $\mathrm{~N}$ & $1 \%$ & $1 \%$ & $4 \%$ & $1 \%$ & $1 \%$ & $5 \%$ \\
\hline
\end{tabular}


A continuación se comentan y ejemplifican cada una de las categorías.

a) Concepto de análisis químico. Este tema es tratado por todos los entrevistados, con mayor o menor detalle, de manera explícita o implícita. Algunos ejemplos son:

Estos temas se dan en una o dos clases teóricas y luego otras clases hacen problemas. Que vean problemas concretos de análisis, por ejemplo, cómo se analiza el acético de un vinagre o un ácido de un fármaco, hay un dossier de problemas de todo tipo: desde determinar el agua oxigenada de una mezcla, y otros. Un poco que ellos vean ejemplos de valoraciones redox, de complejación, de precipitación y de ácido base. De un enunciado en que se dice cómo se hace el análisis y hay unos datos numéricos de la valoración, cómo ellos calculan. (Entrevista G).

Mi experiencia me indica que el primer día de clase les aclare bien, que ellos como ingenieros van a tener que obtener resultados y obtener resultados en una industria generalmente es: "hacer un análisis químico", entonces al análisis químico hay que interpretarlo. Si piden analizar algo tienen que saber que están pidiendo, en cuanto a rapidez del análisis, la exactitud, y además el ingeniero químico, muchas veces, empieza su experiencia profesional en un laboratorio. Tengo una experiencia de 35 años trabajando en la industria y les muestro un esquema que relaciona al Ingeniero Químico, la Planta Industrial, la Investigación y el Laboratorio. (Entrevista V)

En la Unidad 10, por ejemplo se trata: Técnicas de análisis cualitativo por vía húmeda. Análisis cualitativo de cationes: reactivos generales de cationes. Propiedades analíticas y reactivos de identificación. Preparación de la solución para el análisis sistemático de cationes. Marcha sistemática de cationes. Análisis cualitativo de aniones: clasificación analítica de los aniones. Reactivos generales. Propiedades analíticas y reactivos de grupos de aniones. Ensayos previos. Técnica de descarte. (Entrevista $\mathrm{H}$ )

Es una carrera que está orientada a procesos químicos y a petroquímica por la cercanía del Polo Petroquímico, los estudiantes se entusiasman entonces les parece que ingeniería es más la parte de cálculo, toda la parte final, los simuladores, pero luego entienden que esas herramientas del análisis químico las necesitan, no porque las usen a diario como ingenieros, pero tienen que tener el vocabulario para poder entenderse con todo el resto de la gente de una planta y con la gente de laboratorio es fundamental, un ingeniero químico es probable que no tenga que realizar el análisis pero tiene que saber pedirle al laboratorista, al licenciado en química, poder entender lo que el licenciado le dice, esas herramientas las tiene que ver. (Entrevista Q)

La frecuencia de aparición de este tema, remite a la vinculación que todavía parece existir entre el área de conocimiento de la Química Analítica y el antiguo Análisis Químico. Históricamente la primera vez que se utilizó el término Química Analítica fue debido a Fresenius, fundador en 1862 de la primera revista científica totalmente dedicada a esta rama de la Química y que aún se publica, titulada: "Zeitschrift zur Analytische Chemie" es decir, "Revista de Química Analítica", y por otra parte su libro, publicado en 1841, lo tituló "Anleitung zur qualitativen Chemische Analyse" o sea, "Instrucciones para el Análisis Químico cualitativo". Mientras el Análisis Químico es una técnica que nos indica la manera de operar, la Química Analítica es la ciencia elaboradora y creadora de esa técnica; la que establece los principios que justifican el porqué, el cómo de los procesos y la que desarrolla nuevos procedimientos analíticos adecuados a la continua variedad de los objetos de análisis (Arribas-Jimeno, 1985).

La vinculación entre Química Analítica y Análisis Químico, que surge de las entrevistas, es aparente, pues las propuestas metodológicas mencionadas por los entrevistados, así como la bibliografía sugerida o la secuenciación de los temas indican que esta posición ha sido superada ampliamente.

b) Concepto de muestra: este concepto está muy relacionado con el anterior, pues el análisis químico se realiza sobre una porción del material cuyas características se desea conocer, y es de suma importancia que la muestra sea representativa del todo. 
Ellos más tarde, en el mismo curso, van a hacer una práctica de análisis de níquel en una moneda, entonces les digo esto es lo que van a hacer en el laboratorio en unas tres semanas, las prácticas las realizan con otro docente, entonces leemos el proceso analítico, cómo tomamos la muestra, que tratamiento se hace, como determinamos si hay o no interferencias, como las eliminamos, como las enmascaramos, como hacemos la medida, como hacemos los cálculos. Este es un tema que se puede hacer muy práctico, leyendo un análisis real, incluso uno que ellos van a hacer en el laboratorio, para que entiendan que en cualquier análisis hay unas etapas, de modo que ese tema quede claro. (Entrevista G)

Además, les doy un ejemplo, se necesita conocer el grado de contaminación del agua de un lago, en especial nitratos y fosfatos provenientes del lavado de campos fertilizados, en este caso: ¿cómo tomar una muestra representativa del agua del lago? ¿Qué tipo de análisis sería el más adecuado para determinar esas sustancias? ¿Cuál es la concentración máxima permitida para que no afecte al desarrollo de todos los tipos de vida? (Entrevista $\mathrm{V}$ )

En la toma de muestra seguir los pasos que la norma indique, luego entregar la muestra al laboratorio y cuando devuelvan los resultados saber interpretarlos, entonces el ingeniero tiene que ser un interlocutor válido, por ello deben conocer los fundamentos de las técnicas, y como hay muchos instrumentos portátiles, que deben calibrar, tienen lectores digitales, conocer las ventajas y desventajas, las interferencias, no pueden estar mirando un visor y creer que es la verdad revelada, tiene que saber la incerteza del instrumento, la vida útil de un sensor, cómo se calibra, cuáles son las interferencias, cómo va perdiendo la señal. (Entrevista A)

No es que el Ingeniero Químico va a hacer el análisis, puede haber algún caso que sí lo haga, porque para eso, yo pienso, que están los Licenciados en Química y los Técnicos Químicos para hacer tareas de laboratorio, simplemente creo que es importante la entrada y la salida de información, o sea, primero la parte de muestreo y preparación de la muestra de acuerdo a la técnica que se va a usar, y luego interpretar el resultado, los dos extremos tiene que saber hacer el Ingeniero Químico, más que manejar la bureta. (Entrevista B)

c) Ideas acerca de CTSA, estos aspectos han sido abordados, desde diversas perspectivas, por todos los entrevistados. Se detecta una interesante relación con el tema de la contaminación ambiental; el conocimiento de límites de vuelco de efluentes es destacado por algunos de los encuestados como relevante tal como muestran los siguientes párrafos.

Les presento (a los estudiantes) un ejemplo de análisis de un herbicida, extraído del Skoog and West, es un ejemplo muy práctico de como en la vida un químico se encuentra con un problema real de unos animales que se mueren en un parque natural, hacen una hipótesis de qué puede pasar, por ejemplo, puede pasar que el animal haya comido unas hierbas que hayan sido rociadas con un herbicida y ese herbicida tendría arsénico, vamos a ver, vamos a hacer un análisis de arsénico en el animal, entonces plantean cómo lo hacen, que método usan, si usan un método espectroscópico, cómo toman la muestra, cómo la tratan, cómo eliminan interferencias, hay toda una explicación de cuatro o cinco páginas que se las hago leer, les hago las preguntas para que piensen por qué hacen eso, por qué hacen lo otro, y se puede discutir un tema teórico como es el proceso analítico leyendo un ejemplo muy práctico y que ellos de ahí entiendan lo que van a hacer. (Entrevista G)

La Química Analítica para un Ingeniero Químico resulta imprescindible para poder interpretar el resultado de los análisis, para poder evaluar la metodología que van a utilizar, por ejemplo para estudiar un proceso, para determinar una velocidad de reacción, es decir, necesitan saber Química Analítica para interpretar las reacciones químicas, La necesitan también en diseño de reactores y en proyectos de plantas, en gestión ambiental es muy importante en contaminación de los ambientes laborales, es muy importante en la contaminación de las aguas, en contaminación del aire, tienen que tener el conocimiento de Química Analítica. (Entrevista C)

En el tema ambiental eventualmente el ingeniero químico hará el muestreo y seguramente va a estar relacionado con algún de laboratorio donde se realizarán los análisis, tienen que saber a que 
laboratorio va que derivar, conocer cuáles son las técnicas que se pueden usar y tener un idioma que podamos entendernos, por ejemplo, si la muestra es gaseosa se analizará con cromatografía gaseosa, si tengo un sólido lo llevaré a absorción atómica, que el ingeniero tenga un mínimo de criterio aunque no haga personalmente el análisis. (Entrevista A). En la materia Higiene y Seguridad en el Trabajo, se necesita mucho los contenidos de Química Analítica, cuáles son los contaminantes, cómo los van a medir, cuál es el límite de detección del método que se va a usar, asegurarse que están midiendo al valor deseado, dónde van a tomar la muestra, con qué frecuencia. (Entrevista L)

d) También se han encontrado ejemplos en los que se evidencia la vinculación de la Química Analítica con cuestiones sociales. En una de las entrevistas se manifiesta la aplicación de técnicas analíticas a productos biológicos, este hecho relacionaría la notoria responsabilidad ética de los Ingenieros Químicos que trabajan en la Industria de la Alimentación.

Fundamentos de espectroscopia de fluorescencia molecular y su aplicación al análisis de control de calidad de alimentos. (Entrevista H). En el caso de Ingeniería Química, aquí en la Autónoma, se han especializado bastante en la parte de biotecnología o de ingeniería con productos vivos, sería la bioingeniería, son muy expertos aquí en reactores químicos para trabajar con bacterias y con organismos vivos que se dedican a la síntesis de productos, han hecho mucha investigación en esta área con lo cual tienen materias optativas muy específicas de esto. (Entrevista G). Se realizan trabajos prácticos de análisis, por ejemplo, Ácido Acético en vinagre, Calcio en leche y Cloro activo en agua lavandina. (Entrevista $\mathrm{V}$ )

Al comienzo del cuatrimestre se les plantea a los estudiantes que van a tener que realizar un trabajo, elegir un tema, un producto, y buscar en las reglamentaciones vigentes qué especificaciones tienen que cumplir ese producto, y buscar cómo se miden esas características, usar las técnicas y producir un informe. Por ejemplo, el cuatrimestre pasado un grupo de alumnos trabajó con la yerba mate, hasta diseñaron un dispositivo para que cada muestra sea una alícuota correspondiente a cada sorbo de mate, luego sobre cada alícuota realizaban los análisis y evaluaban como iba disminuyendo la concentración de alguno de los componentes a medida que iba lavando la yerba. (Entrevista Z)

Entre los aspectos asociados se encuentra el de Calidad, del análisis de las entrevistas surge que el campo de aplicación de la Química Analítica preferido por los entrevistados resulta ser Control de Calidad. He tenido alumnos que para realizar pasantías le preguntaron si tenía aprobada Química Analítica, más que Química Orgánica o Fisicoquímica, por que empiezan en análisis de laboratorio que siempre en algún proceso de producción hay un análisis de calidad, Química Analítica está relacionada con la calidad, es como que empezando por allí es un aval para comprender el proceso. (Entrevista B)

\section{CONCLUSIONES}

La investigación realizada es de enfoque cualitativo y en particular un estudio de casos, por lo cual no se puede hacer generalizaciones. Sin embargo, es posible arribar a algunas conclusiones respecto a si algunos aspectos sociales y culturales son tratados durante el desarrollo de la asignatura Química Analítica.

El análisis de los programas analíticos no indica la presencia de objetivos relacionados con los aspectos sociales, sólo se ha encontrado detalle de los métodos de análisis químico tradicionales (Volumetría y Gravimetría) e instrumentales (Espectrofotometría, Potenciometría, Conductimetría, Cromatografía). Si bien los aspectos CTSA no son mencionados explícitamente en los mismos, los docentes entrevistados son conscientes de la necesidad de utilizar este enfoque para una adecuada formación de los futuros profesionales. Incluir el enfoque CTSA implica considerar de manera solidaria los aspectos científicos, tecnológicos y éticos. Con los dos primeros aspectos se logrará comprender y abordar los problemas buscando soluciones tecnológicamente aceptables, mientras que el tercero permitirá la realización de una gestión ambiental adecuada según la legislación vigente, respectando la biodiversidad y el uso racional de los recursos. 
En este trabajo se concluye que los aspectos CTSA se presentan como contenidos transversales en las clases de Química Analítica de las Universidades estudiadas. Queda abierta la discusión acerca de una reestructuración curricular de la carrera de Ingeniería Química, en particular de la asignatura Química Analítica, que dé cuenta en forma explícita de los aspectos CTSA y de la responsabilidad profesional del cuidado del ambiente.

\section{REFERENCIAS}

Alvarez-Gayou Jurgenson, Juan. Cómo hacer investigación cualitativa. Fundamentos y metodología. México: Paidos Educador. (2003).

Arribas-Jimeno, S. Introducción a la Historia de la Química Analítica en España. Servicio de Publicaciones de la Universidad de Oviedo. España. (1985).

Corchuelo, M. Una aproximación a los procesos de formación de ingenieros. Revista Electrónica de la Red de Investigación Educativa. ISSN 1794-8061 (en línea), 1(1) (2004). http://revista.iered.org.co Acceso: Agosto 2006.

Glaser, B. y A. Strauss. The Discovery of Grownded Theory. Strategies for Qualitative Research. Weidenfeld and Nicolson, Londres, Inglaterra.(1968).

Grinter, L.E. Summary of the Report on Evaluation of Engineering Education. 1955. Reprinted. Journal of Engineering Education. January, 74-94. (1994).

MIT, Massachusetts Institute of Technology. The Chemical Engineering Profession. United State of America. En: Informe acerca de la profesión del Ingeniero Químico, Consejo de Directores y Decanos de Ingeniería Química. (2008) www.coddiq.es/index2.php Acceso; Marzo 2010.

Speltini, C. y J. Cornejo. Actividades tecnológicas en la formación básica del Ingeniero. Revista Argentina de Enseñanza de la Ingeniería. 6(10), 7-16. Editorial Fundación Universidad Nacional de Río Cuarto. Córdoba, Argentina. (2005).

Stanford University. United States of America. What is Chemical Engineering? En: Informe acerca de la profesión del Ingeniero Químico, Consejo de Directores y Decanos de Ingeniería Química. (2008). www.coddiq.es/index2.php Acceso; Marzo 2010.

University of Surrey-Guildford. Chemical Engineering. United Kingdom. (2009). www2.surrey.ac.uk/ undergraduate/courses/chemicalengineering/ Acceso: Marzo 2010.

University of Aberdeen. Professional Groups: Engineering. United Kingdom. (2009) www.abdn.ac.uk/ engineering/profgrps/chemgrp.php Acceso: Marzo 2010. 